\title{
Goseki histological grading of gastric cancer is an important predictor of outcome
}

\author{
I G Martin, M F Dixon, H Sue-Ling, A T R Axon, D Johnston
}

\begin{abstract}
TNM (tumour, node, metastases) staging has thus far been the most important guide to prognosis in patients with gastric cancer. Histological grading, in contrast, has not provided any additional information. Recently a novel grading system based on tubular differentiation and mucus production has been proposed, which was correlated with patterns of tumour spread found at necropsy. This study set out to assess its value as a determinant of survival after gastric resection. In a consecutive series of 211 patients who had potentially curative resection for gastric cancer, five histological grading systems were assessed: the Lauren type, the WHO type, degree of differentiation, the type of tumour border, and the lymphocytic response to the tumour and compared with the Goseki grading (I-IV). When T and $\mathbf{N}$ stage were taken into account, using Cox's proportional hazards model, only the Goseki grading added further to the ability to predict survival. The proportional hazards ratios were: node negative $v$ node positive $6.5 \mathrm{~T} 1 v \mathrm{~T} 32.45$; Goseki I $v$ Goseki IV 3.1. Five year survival of patients with mucus rich (Goseki II and IV) T3 tumours was significantly worse than that of patients with mucus poor (Goseki I and III) T3 tumours $(18 \%$ v $53 \%, p<0.003)$. Goseki grading identifies subgroups of patients with a poorer prognosis than is predicted by TNM staging alone. It could prove useful in the selection of patients for adjuvant therapy after potentially curative resection for gastric cancer.

(Gut 1994; 35: 758-763)
\end{abstract}

Academic

Departments of

Surgery,

I G Martin

H Sue-Ling

D Johnston

Gastroenterology, A T R Axon

and Pathology, Centre for Digestive Diseases, The General Infirmary The General Infir M F Dixon

Correspondence to: Mr I G Martin, Academic Department of Surgery, The General Infirmary at Leeds, Great George Street, Leeds LS1 3EX.

Accepted for publication 13 October 1993 is widely used, provides useful prognostic information, and is the most important clinical method for staging adenocarcinoma of the stomach. It is widely used in many clinical settings without reference to histological morphology because the latter is felt to be of little practical importance.

It has been recognised for some time logical characteristics and clinical behaviour
Because of the wide variation in the morphological characteristics of gastric cancer, numerous histological classification systems have been proposed. Jarvi ${ }^{2}$ suggested that the histological origin of gastric cancer was within islands of intestinal metaplasia, giving rise to the Lauren classification of gastric cancers into so called intestinal or diffuse types. Gastric cancer has also been classified according to the degree of differentiation exhibited by the tumour. ${ }^{34}$ Ming ${ }^{5}$ proposed a classification based on the histological appearance of the tumour border (infiltrative or expansive). Finally, the WHO proposed a morphological categorisation based on histological appearance and type. ${ }^{6}$ Despite initial enthusiasm, however, for each of these histological classifications of gastric cancer, none has yet added to the TNM staging system in the ability to predict outcome.

Goseki et $a l^{7}$ have recently proposed a novel histological method for classifying gastric cancer based on intracellular mucus production and the degree of tubular differentiation. Four grades of tumour were proposed (Fig 1): group I: tubules well differentiated, intracellular mucus poor; group II: tubules well differentiated, intracellular mucus rich; group III: tubules poorly differentiated, intracellular mucus poor; group IV: tubules poorly differentiated, intracellular mucus rich.

The authors compared this new histological system to the pattern of metastatic spread found at postmortem examination, but its relation to clinical outcome (five year survival) has not yet been reported. We describe here the relation between the Goseki grading system, other histological classification systems, TNM staging, and clinical outcome in a consecutive series of patients who had received potentially curative resection for gastric cancer.

\section{Patients}

Between 1969 and 1990, 517 patients presented to the academic department of surgery with adenocarcinoma of the stomach, of whom $211(41 \%)$ received potentially curative resection (defined as removal of all macroscopic tumour, with clear resection margins). Most were radical $\left(\mathbf{R}_{2}\right)$ resections, with extensive lymphadenectomy. 8 Twenty seven of these 211 patients had intramucosal carcinoma only. These patients were excluded from further analysis because the behaviour of these lesions is well known and any additional prognostic information would be marginal, and in some insufficient pathological material was available for accurate evaluation. In three patients the 

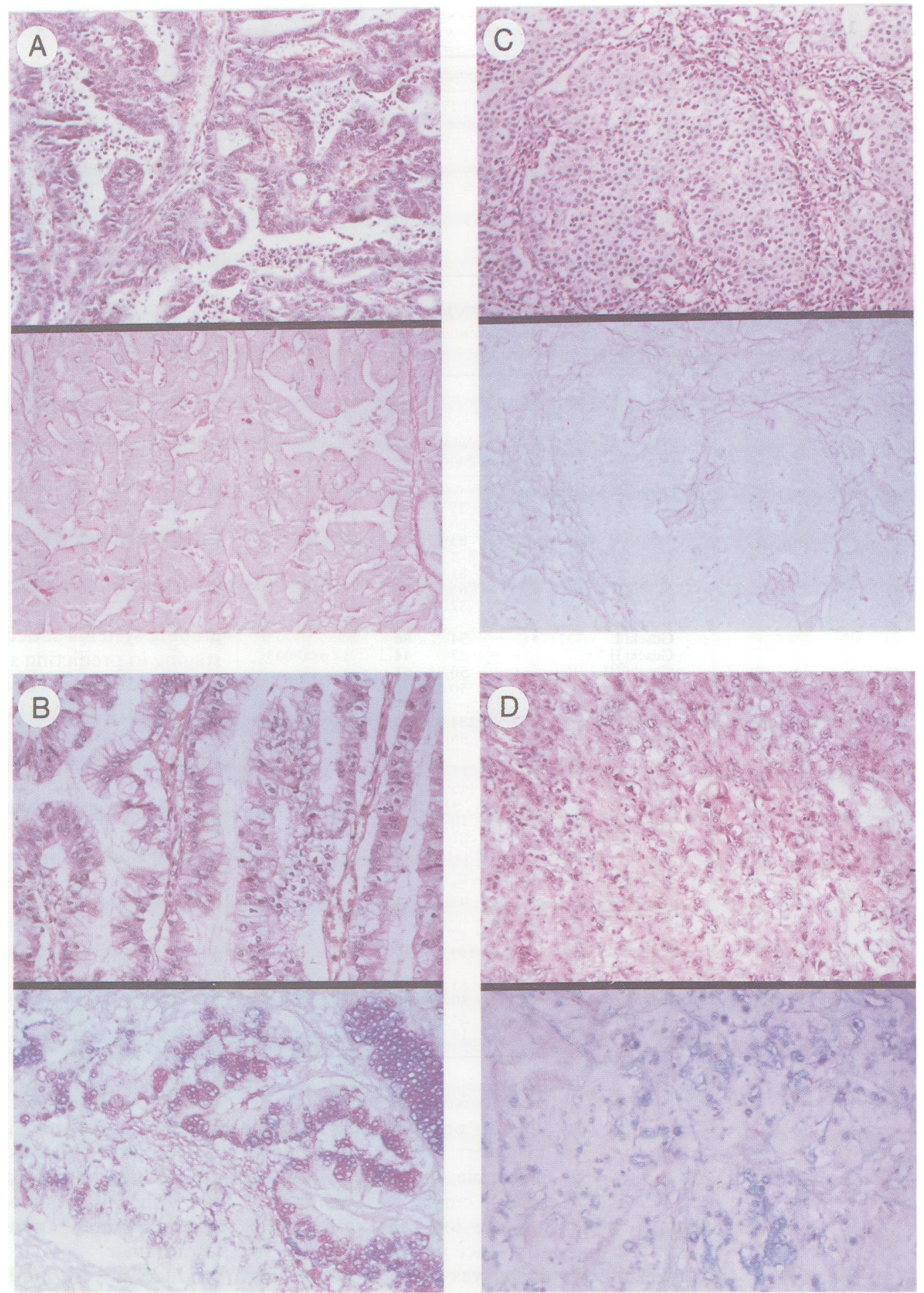

Figure 1: Haematoxylin and eosin (upper panels) and alcian blue PAS stained sections (lower panels) illustrating typical examples of the four Goseki grades: $(A)$ a tumour showing good tubulopapillary differentiation but a poor intracytoplasmic mucin content - grade I; (B) good tubulopapillary differentiation and plentiful intracytopasmic mucin - grade II; (C) poor tubular differentiation and poor mucin content - grade III; (D) poor tubular differentiation but rich mucin content - grade $I V$.

pathological specimen and slides were not available for study. Thus 181 patients were left for analysis. Table I shows the details of these 181 patients.

\section{Methods}

The histological specimens were classified by one of the authors (MFD), without knowledge of the clinical outcome, according to the following systems: Goseki grade (I-IV); Lauren classification (intestinal, mixed, diffuse); WHO classification; Ming classification of the type of border (expansive, infiltrative); degree of differentiation (well, moderate, poor); lymphocyte infiltration of tumour (graded 0-3; no lymphocytes, small numbers, moderate numbers, and numerous lymphocytes).

The tumours were staged using the Unified 1987 TNM system for gastric cancer. ${ }^{5}$ Patients were reviewed every six months at a special gastric follow up clinic. The cause of death in each case was determined from our own records or those of the Yorkshire 
TABLE I Details of the 181 patients who received potentially curative resection for gastric cancer

\begin{tabular}{llllll}
\hline & Goseki I & Goseki II & Goseki III & Goseki IV & Total \\
\hline Male:female & $38: 16$ & $17: 10$ & $33: 17$ & $30: 20$ & $118: 63$ \\
Age (mean) & $68 \cdot 6$ & $67 \cdot 8$ & $66 \cdot 3$ & $65 \cdot 3$ & $66 \cdot 9$ \\
Operation type & 23 & 15 & 17 & 29 & 84 \\
$\quad$ Subtotal gastrectomy: & 27 & 10 & 29 & 18 & 84 \\
$\quad$ Total gastrectomy & 4 & 2 & 4 & 3 & 13 \\
$\quad$ Oesophagogastrectomy & 28 & 7 & 17 & 17 & 69 \\
Tumour stage & 7 & 8 & 11 & 10 & 36 \\
$\quad$ I & 19 & 12 & 22 & 23 & 76 \\
II & & & & & \\
III & & & &
\end{tabular}

TABLE II The influence of TNM staging and histological grading on survival after potentially curative resection for gastric carcinoma

\begin{tabular}{|c|c|c|c|}
\hline Category & $\begin{array}{l}\text { No of } \\
\text { patients }\end{array}$ & $\begin{array}{l}\text { Five year } \\
\text { survival } \\
\text { Kaplan } \\
\text { and Meier } \\
\text { plot (\%) }\end{array}$ & $\begin{array}{l}\text { Significance } \\
\text { log rank } \\
\text { test }\end{array}$ \\
\hline \multicolumn{4}{|l|}{ Unified TNM staging: } \\
\hline $\mathrm{T} 1$ & 35 & 97 & \multirow[t]{4}{*}{$\mathrm{p}<0.001$} \\
\hline $\mathrm{T} 2$ & 60 & 73 & \\
\hline T3 & 83 & 37 & \\
\hline T4 & 3 & - & \\
\hline No & 81 & 88 & \multirow[t]{3}{*}{$\mathrm{p}<0.001$} \\
\hline N1 & 63 & 56 & \\
\hline N2 & 37 & 0 & \\
\hline \multicolumn{4}{|l|}{ Goseki grade: } \\
\hline Goseki I & 54 & 80 & \multirow{4}{*}{$\mathrm{p}<0.003$} \\
\hline Goseki II & 27 & 44 & \\
\hline Goseki III & 50 & 61 & \\
\hline Goseki IV & 50 & 46 & \\
\hline \multicolumn{4}{|l|}{ Lauren classification: } \\
\hline Intestinal & 134 & 65 & \multirow[t]{2}{*}{$\mathrm{p}<0.05$} \\
\hline Diffuse & 36 & 44 & \\
\hline \multicolumn{4}{|l|}{ WHO classification: } \\
\hline Papillary & 11 & 72 & \multirow[t]{5}{*}{-} \\
\hline Tubular & 115 & 66 & \\
\hline Signet ring & 9 & 51 & \\
\hline Mucinous & 19 & 40 & \\
\hline Unclassified & 27 & 42 & \\
\hline \multicolumn{4}{|l|}{ Grade of tumour: } \\
\hline Well differentiated & 32 & 80 & \multirow[t]{3}{*}{$\mathrm{p}<0.05$} \\
\hline Moderately differentiated & 54 & 61 & \\
\hline Poorly differentiated & 95 & 54 & \\
\hline \multicolumn{4}{|l|}{ Ming's classification: } \\
\hline Expansive border & 42 & 73 & \multirow[t]{2}{*}{ NS } \\
\hline Infiltrative border & 139 & 56 & \\
\hline \multicolumn{4}{|l|}{ Lymphocytic infiltration: } \\
\hline Nó lymphocytes & 83 & 56 & \multirow[t]{4}{*}{ NS } \\
\hline Rymphocytes 1 & 50 & 68 & \\
\hline Lymphocytes 2 & 34 & 60 & \\
\hline Lymphocytes 3 & 14 & 56 & \\
\hline
\end{tabular}

Regional Cancer Registry. Follow up was complete in all but one patient. Median follow up was $5 \cdot 1$ years. Survival was calculated by the life table method of Kaplan and Meier. ${ }^{9}$ Survival curves were compared statistically using the log rank test. ${ }^{10}$ The comparative importance of various factors in determining survival was calculated by means of Cox's proportional hazards model. ${ }^{11}$

\section{Results}

HISTOLOGICAL GRADING AND SURVIVAL Among the 181 patients who had potentially curative resection for gastric cancer, operative mortality was $6 \%$. Cumulative survival, calculated by life table analysis, after exclusion of

TABLE III Results of the application of Cox's proportional hazards model

\begin{tabular}{llll}
\hline Parameter & $\beta$ & $e^{\beta}$ & $\begin{array}{l}\text { Example of } \\
\text { hazards ratio }\end{array}$ \\
\hline Nodal involvement N0, N1, N2 & $1 \cdot 41$ & $4 \cdot 1$ & N0 $v$ N2 16.8 \\
$\begin{array}{l}\text { Depth of tumour penetration T1, T2, T3 } \\
\text { Goseki grade I, II, III, IV }\end{array}$ & $0 \cdot 45$ & 1.57 & T1 $v$ T3 2.45 \\
& $0 \cdot 38$ & $1 \cdot 46$ & Goseki I $v$ IV 3.1 \\
\hline
\end{tabular}

operative deaths, was $60 \%$ at five years and $55 \%$ at 10 years.

Table II summarises the relation between lymph node involvement (N), depth of tumour penetration ( $T$ ), and survival. Five year survival in patients without lymph node metastasis was $88 \%$; with lymph node metastasis, $37 \%(\mathrm{p}<0.001)$. Likewise, five year survival of patients with tumour confined to the wall of the stomach (T1 and T2) was $82 \%$, but when the tumour had penetrated through the serosa (T3), survival was only $36 \%(\mathrm{p}<0.001)$.

Table II shows the effects of the various histological grading systems on survival, irrespective of $\mathrm{T}$ and $\mathrm{N}$ staging. The Goseki grade, Lauren type, and grade of tumour were found to be important predictors of outcome when analysed without account of $T$ and $N$ stage. The WHO classification was not designed to predict clinical outcome and most tumours fall in just one category (tubular); it is therefore inappropriate to apply statistical tests across the various categories.

To find out if any of these histological grading systems added further to the $\mathrm{T}$ and $\mathrm{N}$ staging in predicting survival, the results were analysed by means of Cox's proportional hazards model. The histological factors that seemed to be independently associated with survival after potentially curative resection for gastric cancer (see Table II) were placed in the model together with $\mathrm{T}$ and $\mathrm{N}$ stage. After the first analysis, non-contributory factors $(p>0.05)$ were removed in a stepwise fashion. The final model contained only three factors: nodal involvement ( $N$ stage), depth of tumour penetration ( $T$ stage), and the Goseki grade of the tumour. Table III shows the results of this analysis.

Figure 2 shows the relation between the Goseki histological grading system and clinical outcome. Five year survival was $80 \%$ in patients with Goseki I tumours (tubules well differentiated, intracellular mucus poor) but only $46 \%$ in patients with Goseki IV tumours (tubules poorly differentiated, intracellular mucus rich) $(\mathrm{p}<0.001)$.

Of the two components of the Goseki system, namely tubular differentiation and intracellular mucus production, mucus production was found to be the more important determinant of clinical outcome. Five year survival of patients with tumours that showed good tubular differentiation (Goseki I and II) was $69 \%$, compared with $54 \%$ in patients with tumours that showed poor tubular differentiation (Goseki III and IV) $(p<0 \cdot 05)$. In contrast, five year survival of patients with mucus poor tumours (Goseki I and III) was $71 \%$, but only $45 \%$ in patients with mucus rich tumours (Goseki II and IV) $(p<0.001)$. The effect of mucus production on survival was also found to be independent of $\mathrm{T}$ and $\mathrm{N}$ staging (Figs 3 and 4 ).

\section{Discussion}

The American pathologist Stout commented in 1953 that 'histological classification was 


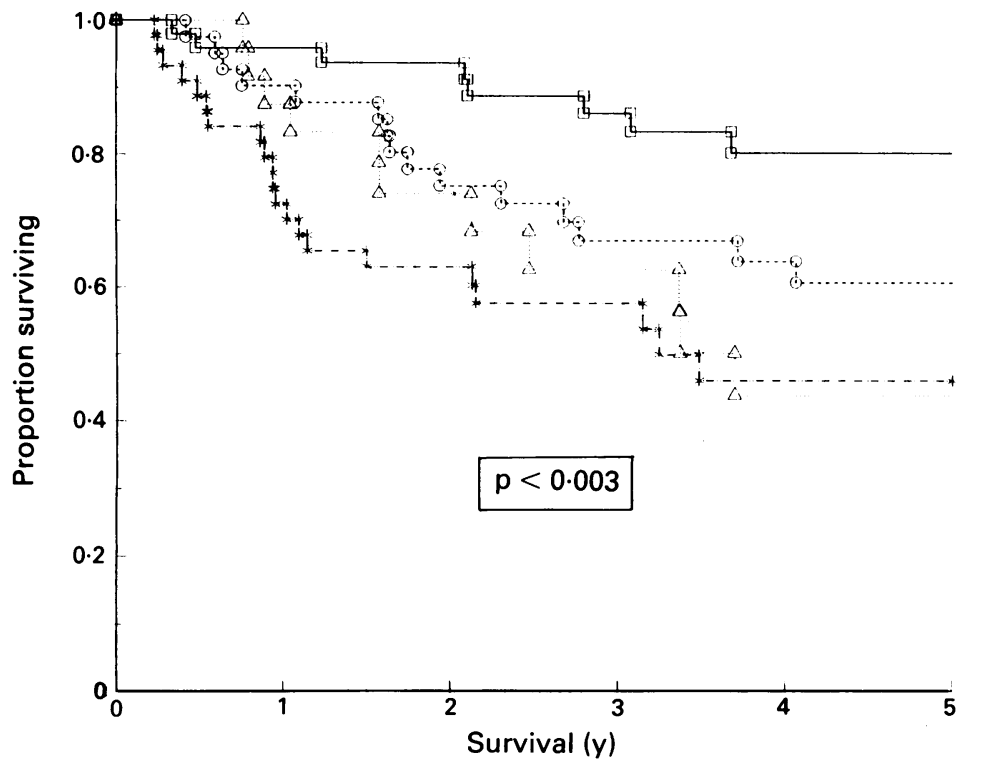

Figure 2: Survival after potentially curative resection for gastric cancer according to the Goseki grade of the tumour.

valueless and that a knowledge of the gross appearance and spread of tumours was of more value in assessment of prognosis'. ${ }^{12}$ This view has persisted for many years and there is widespread belief among clinicians and pathologists that histological grading of gastric cancers adds nothing further to the assessment of prognosis over and above what may be derived from the TNM stage of the tumour. This report is the first description of a histological grading system that shows substantial and statistically significant differences in survival among patients with gastric cancer, after tumour stage has been taken into account. Indeed we found that the Goseki histological grade was as important as the depth of tumour penetration ( $T$ stage) in predicting outcome using Cox's proportional hazards model.

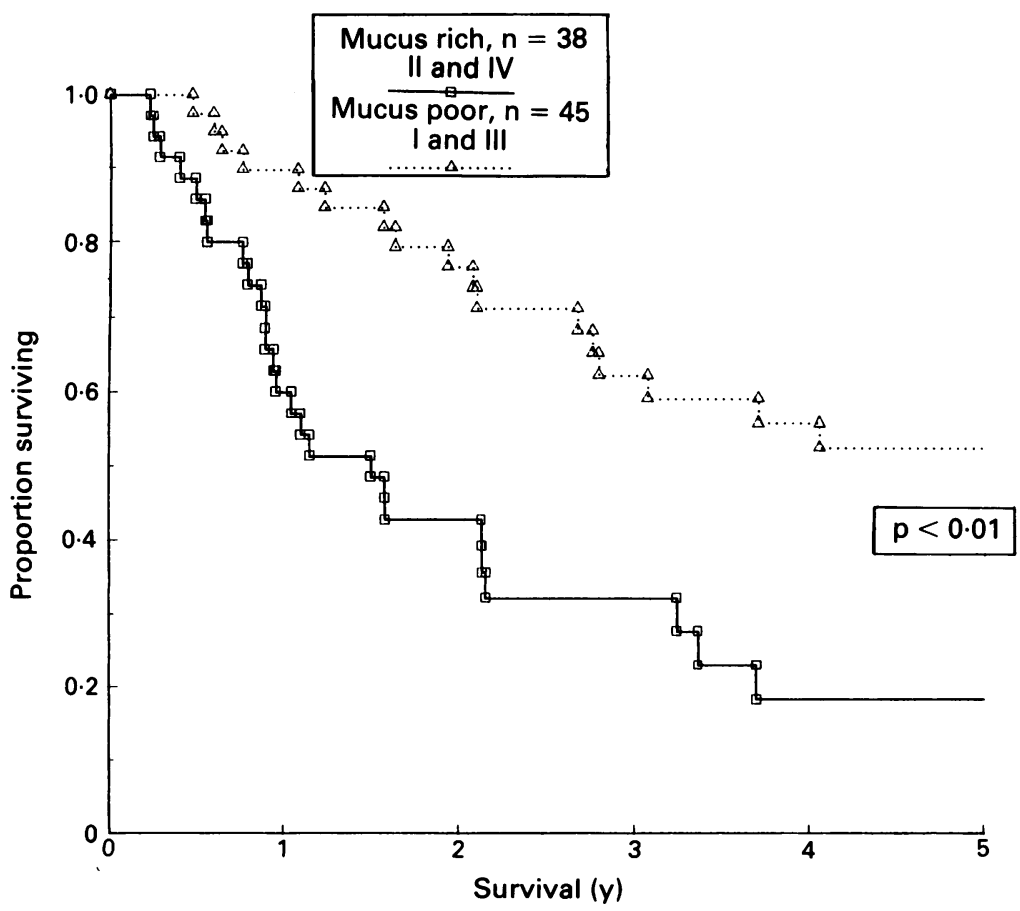

Figure 3: Survival after potentially curative resection for 83 patients with T3 gastric cancer according to mucus production.
Goseki et al based their system on two features of gastric cancer: the structural features of tubular differentiation and the functional state of mucus production. They stated that few would challenge the use of tubular differentiation, but believed that the use of mucus production would be questioned by others. We have shown, however, that degree of mucus production by the tumour is of greater importance than tubular differentiation in the assessment of prognosis. We therefore support Goseki's opinion that accumulation of mucus does not merely represent the absence of a suitable tubular structure for mucus excretion.

In Goseki's original paper the distribution of tumours among the four grades was different to that in our series with fewer patients in Goseki grade I and proportionately more in Goseki grade IV. Although the reason for this is not absolutely clear there are two possible explanations; firstly the fact that Goseki's study was carried out on necropsy material and hence more likely to include patients with aggressive tumours and secondly it is well recognised that there is a higher proportion of diffuse poorly differentiated tumours in Japan.

The Lauren classification has been one of the most widely applied histological grading systems, particularly in epidemiological studies. While this system may have its applications in such broad epidemiological studies, there is no evidence that the Lauren classification can add to the prognostic information that is provided by TNM staging. In the original description of the Lauren system there was an eight per cent difference in survival between the intestinal and diffuse groups at three years $(43 \% \quad v \quad 35 \%$ respectively). ${ }^{13}$ When the Lauren classification was applied, however, by other authors to larger series of patients, no difference in five year survival was found. ${ }^{14} \mathrm{~A}$ further disadvantage of the Lauren system is that between 10 and $20 \%$ of cases remain unclassified. Ming's system, ${ }^{4}$ which is based on tumour infiltration, has the advantage over the Lauren system that all cases can be classified, but again there is no evidence that it is of prognostic value. While the WHO classification $^{3}$ is widely used, it has several drawbacks; for example, it fails to classify a significant proportion of tumours and many tumours straddle two or more categories. Again, there is little evidence that the WHO classification is important in determining clinical outcome, not unsurprising when it is realised that this was not the reason for the formulation of this system.

There is no evidence in published works that any of the other histological grading systems is useful in predicting outcome, once tumour spread (TNM stage) has been considered, a view that is supported by our findings. It was disappointing to find that the degree of lymphocyte infiltration of the tumour had no apparent effect on survival. It would be satisfying if it could be shown that the body's own immune response could be shown to have an effect on clinical outcome. This is in contrast 


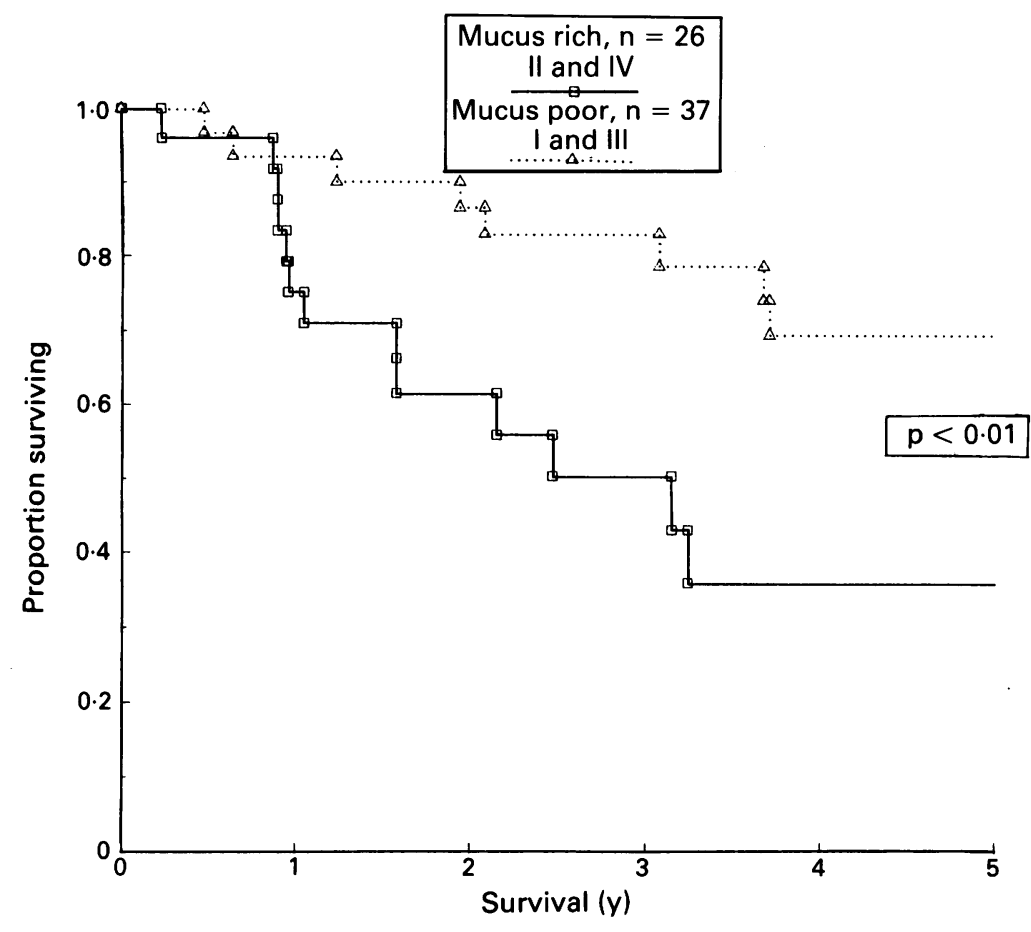

Figure 4: Survival after potentially curative resection for 63 patients with N1 gastric cancer according to mucus production. containing little mucus, although this must be interpreted with caution given that it was a postmortem study. One research group has shown chromosome 1 abnormalities in some patients with gastric cancer, ${ }^{19}$ in an area known to code for mucus production. Whether tumours that are rich in intracellular mucus have different underlying molecular biological abnormalities compared with tumours poor in mucus remains to be determined.

We would propose that the Goseki classification, if it is to be widely adopted, should be changed, to transpose grades II and III, so as to reflect the greater importance of mucus production on survival. Thus, our proposed modification of the system would be as follows: group I: intracellular mucus poor, tubules well differentiated; group II: intracellular mucus poor, tubules poorly differentiated; group III: intracellular mucus rich, tubules well differentiated; group IV: intracellular mucus rich, tubules poorly differentiated.

It is not clear whether these findings are of any clinical relevance. At present, we feel that this histological grading system could not be applied to preoperative endoscopic biopsy specimens with any degree of accuracy, because of the small samples of tissue available, although we have not formally tested this ourselves. Nevertheless, we think that the Goseki classification, or our proposed modification of it, is possibly of use in helping to select patients for adjuvant treatment after surgical resection because it identifies groups of patients in whom the prognosis is significantly worse than that predicted by TNM staging alone. We are conscious, however, that the analysis presented here is retrospective and based on one pathologist's assessment of the histology. Whether this staging system is valid when tested prospectively and whether it is both repeatable and reproducible remains to be ascertained.

In summary, we have shown that the new histological grading system proposed by Goseki provides a more accurate prognosis in patients with gastric carcinoma when added to the TNM staging system alone. In this, it differs from all other histological grading systems previously described. Mucus production by the tumour was found to be more important in determining patients' survival than the degree of tubular differentiation. If the Goseki system is to be widely adopted, we would propose that grades II and III be transposed to place greater emphasis on the prognostic importance of mucus production.

1 Kennedy BJ. The unified international gastric cancer staging classification. Scand $\mathcal{f}$ Gastroenterol 1987; 22 (suppl 133): 1-13.

2 Jarvi O, Lauren P. On the role of the heterotopias of the intestinal epithelium on the pathogenesis of gastric cancer. Acta Pathol Microbiol Scand 1951; 29: 26-44.

3 Hirota T, Itabashi M, Suzuki K, Yoshida S. Clinicopathologic study of minute and small early gastric cancer. Pathol Anmu 1980; 15: 1-19.

4 Ming SC. Gastric carcinoma: A pathological classification. Cancer 1977; 39: 2475-85.

5 Kato Y, Kitigawa T, Nakamura K, Sugano H. Changes in the histological types of gastric carcinoma in Japan. Cancer 1981; 48: 2084-7. intracellular mucus. There is some evidence from Goseki's paper that the tumours rich in mucus were associated with greate numbers of lymph node metastasis than those 
6 Oota K, Sobin LH. International histological classification of tumours No 18: histological typing of gastric and of tumours No 18: histological typing of

7 Goseki N, Takizawa T, Koike M. Differences in the mode of extension of gastric cancer classified by histological type: new histological classification of gastric carcinoma Gut 1992; 33: 606-12.

8 Japanese Research Society for Gastric Cancer. The general rules for the gastric cancer study in surgery and pathology. Fpn f Surg 1981; 11: 127-45.

9 Kaplan EL, Meier P. Non-parametric estimation from incomplete observations. F Am Stat Assoc 1958; 53: 457-81.

10 Peto R, Pike MC, Armitage P, Breslou NE, Cox DR, Howard SV, et al. Design and analysis of randomised clinical trials requiring prolonged observation of each patient. II Analysis and examples. Br $\mathcal{f}$ Cancer $1977 ; 35: 1-39$.

11 Cox DR. Regression models and life tables. $\mathcal{F} R$ Statist Soc 1972; 34: 187-202.

12 Stout AP. Armed forces institute of pathology: F65 tumours of the stomach, fasicle 21. Washington, DC: 1953.
13 Lauren $P$. The two histological main types of gastric carcinoma. Acta Pathol Micobiol Scand 1965; 64: $31-49$.

14 Hawley PR, Westerman P, Morson BC. Br F Surg 1970; 57: $877-83$.

15 Schachenmayr W, Haferkamp O. Prognostic significance of stromal reaction in gastric carcinoma. In: Herfarth of stromal reaction in gastric carcinoma. In: Herfarth
$\mathrm{Ch}$, Sclag P, eds. Gastric cancer. Berlin: Springer Verlag, 1979: 182-92.

16 Paile A. Morphology and prognosis of carcinoma of the stomach. Ann Chir Gynaecol Fenn 1971; 60 (suppl 175): $1-56$.

17 Brander WL, Needham PRG, Morgan AG. Indolent mucoid carcinoma of stomach. F Clin Pathol 1974; 27 $536-41$

18 Ishii T, Ikegami N, Hosada Y, Koide O, Kanero M. The biological behaviour of gastric cancer. F Pathol 1981; 134: 97-115.

19 Fey MF, Hesketh C, Wainscoat JS, Gendler S, Thein SL. Clonal allele loss in gastrointestinal cancers. Br $\mathcal{f}$ Cancer 1989; 59: 750-4. 